This item was submitted to Loughborough's Research Repository by the author.

Items in Figshare are protected by copyright, with all rights reserved, unless otherwise indicated.

\title{
Structural relaxation in PLLA: Contribution of different scale motions
}

PLEASE CITE THE PUBLISHED VERSION

https://doi.org/10.1016/j.tca.2018.12.027

PUBLISHER

(c) Elsevier

VERSION

AM (Accepted Manuscript)

PUBLISHER STATEMENT

This paper was accepted for publication in the journal Thermochimica Acta and the definitive published version is available at https://doi.org/10.1016/j.tca.2018.12.027

\section{LICENCE}

CC BY-NC-ND 4.0

\section{REPOSITORY RECORD}

Righetti, Maria C., and Elisa Mele. 2019. "Structural Relaxation in PLLA: Contribution of Different Scale Motions". figshare. https://hdl.handle.net/2134/36686. 


\title{
Structural Relaxation in PLLA: contribution of different scale motions
}

\author{
Maria Cristina Righetti *a and Elisa Mele ${ }^{\mathrm{b}}$

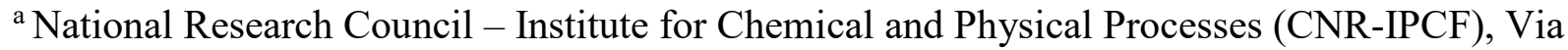 \\ Moruzzi 1, 56124 Pisa, Italy. E-mail: cristina.righetti@pi.ipcf.cnr.it \\ ${ }^{\mathrm{b}}$ Materials Department, Loughborough University, LE11 3TU, Loughborough, UK
}

\begin{abstract}
The enthalpy relaxation in amorphous and semi-crystalline poly(L-lactic acid) (PLLA) has been investigated at low and high undercooling to identify the different scale motions that contribute to physical ageing, and the temperature ranges in which they are active. The temperature limit below which exclusively small-scale localized motions produce structural relaxation in PLLA has been identified. In addition, motions different from pure cooperative large-scale motions and pure smallscale local rearrangements, localized in the MAF, are found to mainly contribute to structural relaxation at intermediate undercooling. These motions are suggested to be related with the JohariGoldstein process.
\end{abstract}

Keywords: Mobile amorphous fraction, Rigid amorphous fraction, Structural relaxation, Cooperative motions, Local motions

\section{Introduction}

The amorphous phase of semi-crystalline polymers, as poly(L-lactic acid) (PLLA) is constituted by a mobile fraction (MAF), which vitrifies and devitrifies at the bulk $T_{g}$, and a rigid fraction (RAF), located at the amorphous/crystal interface, which vitrifies and devitrifies at temperatures higher than the bulk $T_{g}[1]$. Both MAF and RAF contribute to structural relaxation upon annealing at temperatures below $T_{g}$ [2]. It was recently demonstrated that for amorphous and semi-crystalline PLLA, the enthalpy recovered after isochronal annealing $\left(\Delta h_{a}\right)$ at different annealing temperatures $\left(T_{a}\right)$ exhibits a markedly asymmetric bell-shape when plotted as a function of the undercooling $\left(T_{g}-T_{a}\right)$, with a pronounced tail on the high $\left(T_{g}-T_{a}\right)$ side [2]. For the semi-crystalline samples, the $\Delta h_{a}$ values normalized to the respective mobile amorphous weight fraction, were found to diverge progressively from the amorphous curve by increasing the undercooling [2]. This behaviour was rationalized by 
assuming that also RAF takes part in the enthalpy relaxation, with a contribution that becomes recognizable at high undercooling. RAF does not take part into the cooperative segmental motions around $T_{g}$, and can participate in the structural relaxation only through small-scale conformational rearrangements, which are barely influenced by the confining crystalline regions [3-6].

In this work, the study of the enthalpy relaxation in amorphous and semi-crystalline PLLA is extended to high $\left(T_{g}-T_{a}\right)$ values, to identify the different scale motions that contribute to physical ageing, and the temperature ranges in which they are active

\section{Experimental Section}

PLLA homopolymer (0 \% D-lactic acid units) was purchased from Polysciences Inc, U.S.A. The mass-average molar mass $\left(M_{w}\right)$ was approximately $100,000 \mathrm{~g} \mathrm{~mol}^{-1}$. To avoid degradation of the samples during thermal treatment at high temperatures, PLLA was dried in a vacuum oven at $60{ }^{\circ} \mathrm{C}$ for $24 \mathrm{~h}$ before use.

A PerkinElmer Differential Scanning Calorimeter DSC 8500, equipped with an IntraCooler III as refrigerating system, was utilized. The instrument was calibrated in temperature with high purity standards materials (indium, naphthalene, cyclohexane) at zero heating rate, according to the procedure for conventional DSC [7].

The PLLA samples were heated from room temperature to $473 \mathrm{~K}$ at a rate of $50 \mathrm{~K} \mathrm{~min}^{-1}$, and maintained at this temperature for $2 \mathrm{~min}$. Subsequently, the samples were either quenched at $323 \mathrm{~K}$ and immediately reheated to $368 \mathrm{~K}$ at $300 \mathrm{~K} \mathrm{~min}^{-1}$, to permit cold-crystallization into $\alpha^{\prime}$-modification (crystallization time: $90 \mathrm{~min}$ ), or cooled from $473 \mathrm{~K}$ to $408 \mathrm{~K}$ at $300 \mathrm{~K} \mathrm{~min}^{-1}$, to allow meltcrystallization into $\alpha$-form (crystallization time: $130 \mathrm{~min}$ ) [8]. At the end of the crystallization, the samples were quenched at $293 \mathrm{~K}$. A completely amorphous sample was also obtained by rapid cooling from $473 \mathrm{~K}$ to $293 \mathrm{~K}$. The amorphous and semi-crystalline samples were immediately heated from $293 \mathrm{~K}$ to $473 \mathrm{~K}$ at $10 \mathrm{~K} \mathrm{~min}^{-1}$, in order to register the apparent specific heat capacity $\left(c_{p, a p p}\right)$ curves, and thus measure the $T_{g}$ and the amorphous content.

The $T_{g}$ values were determined as fictive temperature for all the samples [9], which is approximately equivalent to the $T_{g}$ upon cooling [10]. The weight fraction of the mobile amorphous portion ( $w_{M A F}$ ) was determined at $T_{g}$ from the specific heat capacity increment, divided by the $\Delta c_{p}$ of the completely amorphous sample; whereas the crystalline weight fraction $\left(w_{C}\right)$ was determined from the area of the exothermal heat flow rate signal detected during the isothermal crystallizations at 368 $\mathrm{K}$ and $408 \mathrm{~K}$, divided by the enthalpy of melting of $100 \%$ crystalline $\alpha^{\prime}$ - and $\alpha$-forms [11]. Finally, the rigid amorphous weight fraction $\left(w_{R A F}\right)$ was calculated by difference. 
The $T_{g}$ and the specific heat increment at $T_{g}\left(\Delta c_{p}\right)$ for the amorphous and semi-crystalline PLLA samples, together with the respective $w_{C}, w_{M A F}$ and $w_{R A F}$ values are reported in Table 1 . The different glass transition temperatures of PLLA after crystallization at low and high $T_{c}$ s were connected to the vitrification/devitrification of a slightly constrained and unconstrained mobile amorphous fractions, respectively [12].

Table 1. $T_{g}$ and specific heat capacity increment at $T_{g}\left(\Delta c_{p}\right)$, crystalline $\left(w_{C}\right)$, mobile amorphous $\left(w_{M A F}\right)$ and rigid amorphous $\left(w_{R A F}\right)$ weight fractions of the amorphous and semi-crystalline PLLA samples investigated.

Estimated error: $\pm 0.5 \mathrm{~K}$ for $T_{g}, \pm 0.02 \mathrm{~J} \mathrm{~g}^{-1} \mathrm{~K}^{-1}$ for $\Delta c_{p}, \pm 0.02$ for $w_{C}$ and $w_{M A F}, \pm 0.04$ for $w_{R A F}$.

\begin{tabular}{cccccc}
\hline PLLA & $\begin{array}{c}T_{g} \\
(\mathrm{~K})\end{array}$ & $\begin{array}{c}\Delta c_{p} \text { at } T_{g} \\
\left(\mathrm{~J} \mathrm{~g}^{-1} \mathrm{~K}^{-1}\right)\end{array}$ & $w_{C}$ & $w_{M A F}$ & $w_{R A F}$ \\
\hline amorphous & 332.0 & 0.56 & - & 1.00 & - \\
$T_{c}=368 \mathrm{~K}$ & 342.0 & 0.20 & 0.36 & 0.38 & 0.26 \\
$T_{c}=408 \mathrm{~K}$ & 339.5 & 0.15 & 0.43 & 0.28 & 0.29
\end{tabular}

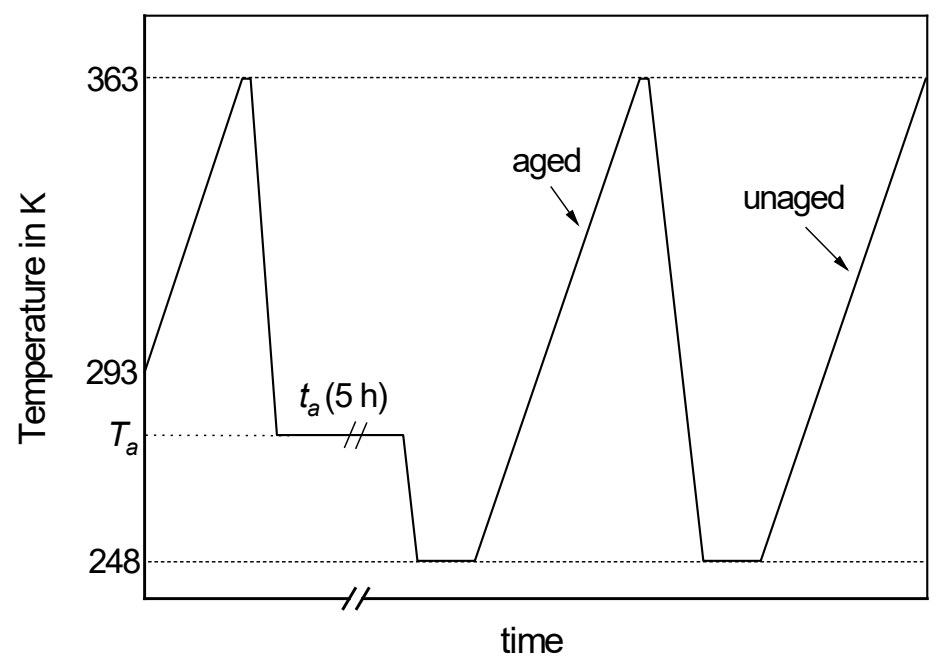

Fig.1 Temperature profile for the enthalpy relaxation experiments

The study of the enthalpy relaxation at different $T_{a}$ s for a fixed ageing time was performed according to the temperature profile shown in Fig. 1. The semi-crystalline PLLA samples were heated to $363 \mathrm{~K}$, kept for $0.3 \mathrm{~min}$ at $363 \mathrm{~K}$, and cooled at the fastest available cooling rate to the preselected annealing temperature $T_{a}$ (average cooling rate: $90 \mathrm{~K} \mathrm{~min}^{-1}$ ), included between $281 \mathrm{~K}$ and $341 \mathrm{~K}$. The samples were maintained at $T_{a}$ for $t_{a}=5$ hours, then cooled down to $248 \mathrm{~K}$ at the fastest cooling rate 
(average cooling rate: $\left.60 \mathrm{~K} \mathrm{~min}^{-1}\right)$. The $c_{p, \text { app }}$ curves of the aged samples ( $c_{p \text {,app,aged }}$ ) were obtained by heating at $20 \mathrm{~K} \mathrm{~min}^{-1}$ from $248 \mathrm{~K}$ to $363 \mathrm{~K}$, whereas the $c_{p \text {,app }}$ curves of the unaged samples ( $\left.c_{p \text {,app,unaged }}\right)$ were achieved, after cooling from $363 \mathrm{~K}$ to $248 \mathrm{~K}$ at the highest cooling rate, by reheating from $293 \mathrm{~K}$ to $363 \mathrm{~K}$ at $20 \mathrm{~K} \mathrm{~min}^{-1}$. For the amorphous PLLA, $T_{a}$ was between $273 \mathrm{~K}$ and $331 \mathrm{~K}$, with the limit temperature of $363 \mathrm{~K}$ reduced to $353 \mathrm{~K}$ to prevent crystallization of the sample.

\section{Results and discussion}

In order to illustrate the evolution of the apparent specific heat capacity curves after annealing of 5 hours at different $T_{a} \mathrm{~s}$, some selected $c_{p \text {,app,aged }}$ and the corresponding $c_{p \text {,app,unaged }}$ for amorphous PLLA are displayed in Figure 2. The difference between the $c_{p \text {,app,aged }}$ and $c_{p \text {,app,unaged }}$ curves for amorphous and semi-crystalline PLLA samples $\left(\Delta c_{p, \text { app }}=c_{p, \text { app,aged }}-c_{p, \text { app } \text {,unaged }}\right)$, after annealing at different $T_{a}$ s for 5 hours, was thus derived. Theses $\Delta c_{p, a p p}$ curves are shown in Figure 3.
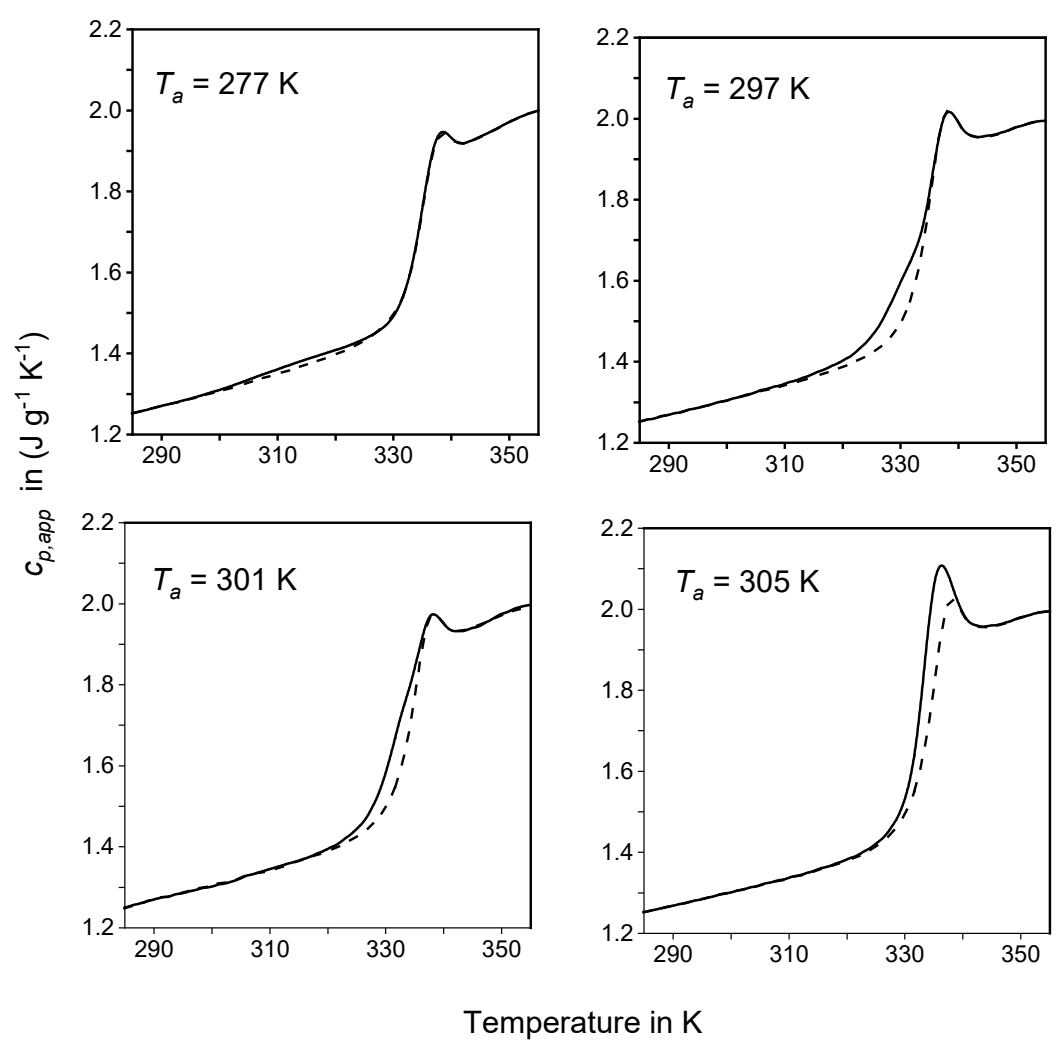

Fig. 2 Apparent specific heat capacity $\left(c_{p, a p p}\right)$ curves of aged ( $c_{p, a p p, a g e d}$, solid line) and unaged $\left(c_{p, a p p, \text { unaged, }}\right.$ dashed line) amorphous PLLA upon heating at $20 \mathrm{~K} \mathrm{~min}^{-1}$, after annealing of 5 hour at the indicated $T_{a} \mathrm{~s}$. 
The enthalpy that relaxes during the annealing of a glass at $T_{a}$ for a period of time $t_{a}, \Delta h_{a}\left(T_{a}\right.$, $t_{a}$ ), recovered during the successive heating run, is determined by integration of the $\Delta c_{p, a p p}$ curve, according to the following relation [13]:

$$
\Delta h_{a}\left(T_{a}, t_{a}\right)=\int_{T_{1}}^{T_{2}} \Delta c_{p, a p p} d T
$$

where $T_{1}$ and $T_{2}$ are two temperatures well below and above the glass transition region respectively. In Fig. 2 the $\Delta c_{p, a p p}$ curves registered after annealing at the lowest investigated $T_{a} \mathrm{~s}$ are highlighted in red, as better discussed below.
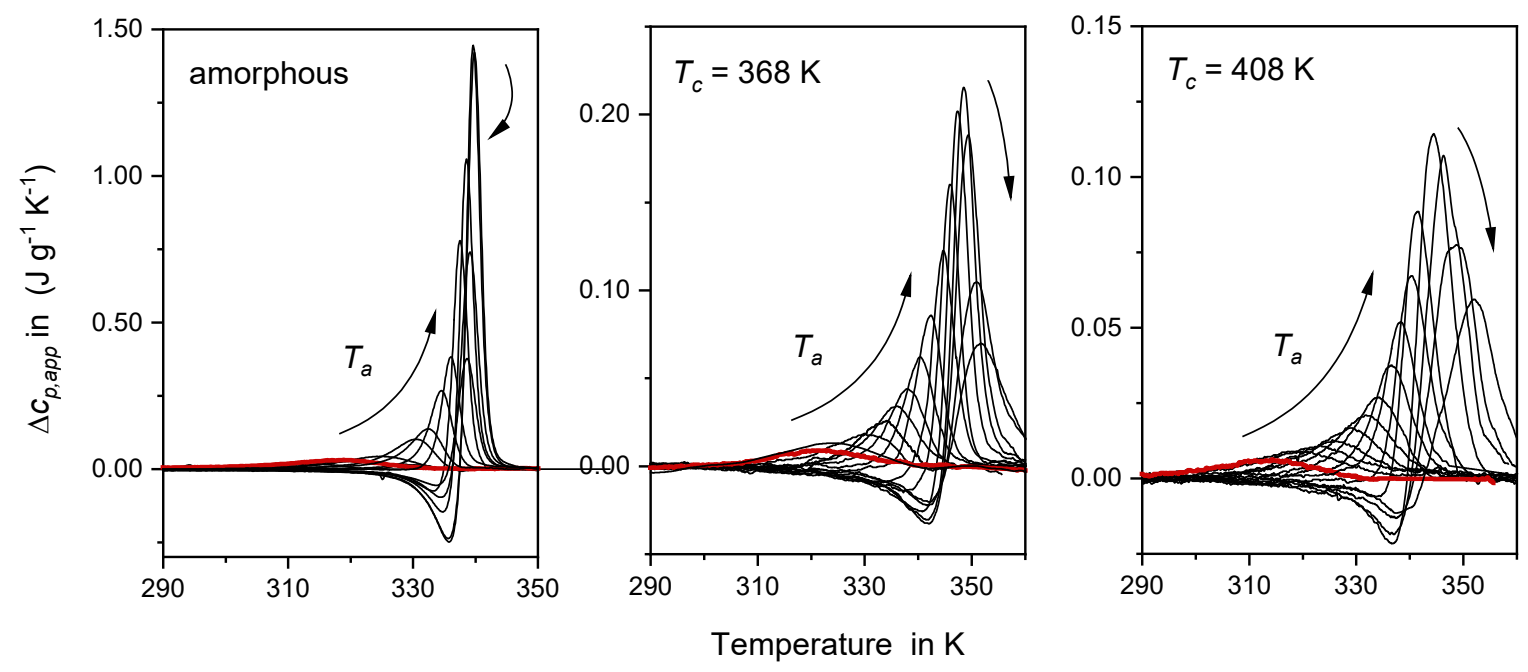

Fig. $3 \Delta c_{p, a p p}$ curves of amorphous and semi-crystalline PLLA crystallized at the $T_{c}$ s indicated after annealing of 5 hour at different $T_{a}$ s. The red solid lines are the $\Delta c_{p, a p p}$ curves after annealing at the lowest $T_{a} \mathrm{~s}$.

Fig. 4(A) displays the enthalpy recovered, $\Delta h_{a}$, as a function of the undercooling $\left(T_{g}-T_{a}\right)$, whereas Fig. 4(B) and 4(C) show the trends of $\Delta h_{a}$ after normalization with respect to the overall non-crystalline fraction $\left(1-w_{C}\right)=\left(w_{M A F}+w_{R A F}\right)$, and the mobile amorphous fraction $w_{M A F}$, respectively. These curves are almost coincident with those reported in the former study up to the undercooling of about $45 \mathrm{~K}$ [1], although a slightly different thermal protocol was here used. Fig. 4(A) reports also the maximum enthalpy, $\Delta h_{a, \max }$, that can be lost by the amorphous and semicrystalline PLLA samples at a given $T_{a}$, estimated as $\Delta c_{p} \cdot\left(T_{g}-T_{a}\right)$, where $\Delta c_{p}$ is the specific heat capacity increment at $T_{g}$. 

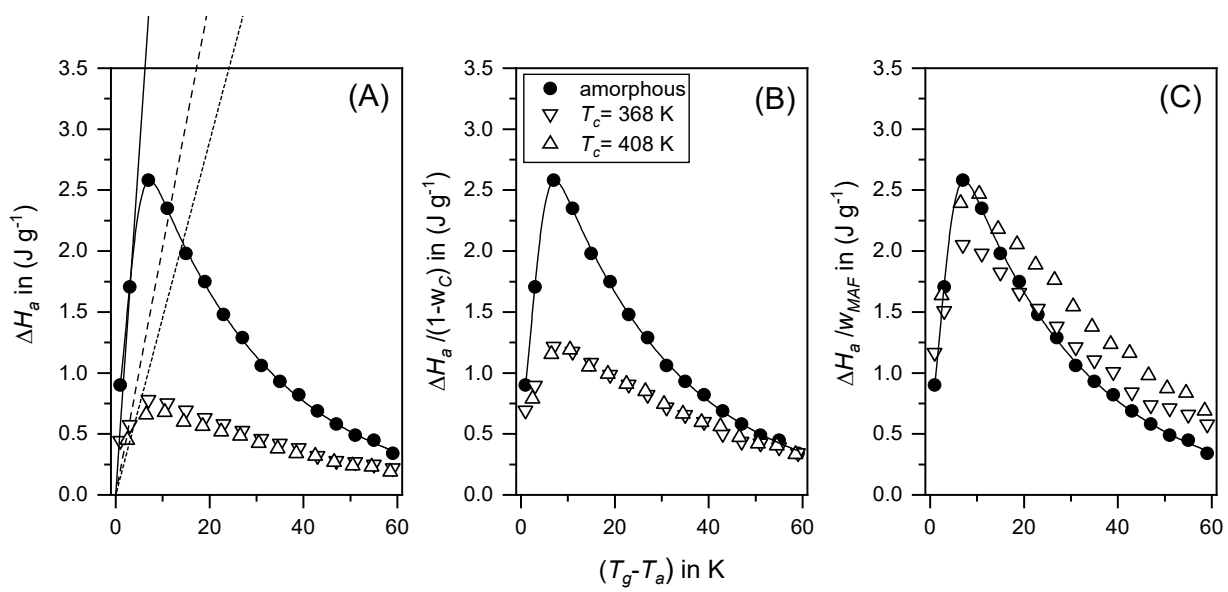

Fig. 4 (A) Reduction in enthalpy $\left(\Delta h_{a}\right)$, (B) reduction in enthalpy normalized to the total amorphous fraction $\left[\Delta h_{a} /\left(1-w_{C}\right)\right],(\mathrm{C})$ reduction in enthalpy, normalized to the mobile amorphous fraction $\left(\Delta h_{a} / w_{M A F}\right)$, after 5 hours of annealing for amorphous and semi-crystalline PLLA samples, as a function of $\left(T_{g}-T_{a}\right)$. The solid line that connects the $\Delta h_{a}$ data of the amorphous PLLA is a guide to the eye. The solid, dashed and dotted lines are the maximum enthalpy that can be lost at a given $\left(T_{g}-T_{a}\right)$ by the amorphous and semi-crystalline PLLA crystallized at $368 \mathrm{~K}$ and $408 \mathrm{~K}$, respectively. Estimated error for $\Delta h_{a}: \pm 0.1 \mathrm{~J} \mathrm{~g}^{-1}$, for $\left[\Delta h_{a} /\left(1-w_{C}\right)\right]$ and $\left(\triangle h_{a} / w_{M A F}\right): \pm 0.15 \mathrm{~J} \mathrm{~g}^{-1}$.

The main finding discussed in the previous work regards the deviation of the $\Delta h_{a} / w_{M A F}$ data from the amorphous curve at high undercooling. The overestimation of the role of the MAF was considered as a proof that the RAF, which is absent in the amorphous PLLA, also takes part in the enthalpy relaxation. It was suggested that the RAF contribution becomes recognizable with reducing $T_{a}$, in parallel with the concomitant decrease in the cooperative segmental motions, which are active during physical ageing only in the MAF regions and primarily at low undercooling [2].

However, the temperature ranges in which the different motions predominantly contribute to the structural relaxation of PLLA were not identified. From this point of view, the present Fig. 4(B) appears very significant, because it reveals that at undercooling higher than $55 \mathrm{~K}$, the relaxed enthalpy normalized to the total non-crystalline fraction $\left[\Delta h_{a} /\left(1-w_{C}\right)\right]$ overlaps the amorphous curve. This means that MAF and RAF contribute similarly to structural relaxation at $\left(T_{g}-T_{a}\right)>55 \mathrm{~K}$. This can occur exclusively through localized motions, the only ones active in the rigid amorphous fraction at temperatures below $T_{g}$ [3-6]. The matching of the $\Delta h_{a}$ and $\left[\Delta h_{a} /\left(1-w_{C}\right)\right]$ curves for the amorphous and semi-crystalline samples at $\left(T_{g}-T_{a}\right)>55 \mathrm{~K}$ indicates that also in amorphous PLLA, structural relaxation originates from local motions at this undercooling. Secondary $\beta$-relaxations were indeed found active in amorphous and semi-crystalline PLLA down to about $-100{ }^{\circ} \mathrm{C}[5,14,15]$.

Figure 3 displays that with reducing $T_{a}$, the $\Delta c_{p \text {,app }}$ peaks shifts toward temperatures progressively lower. After annealing at the lowest $T_{a} \mathrm{~s}$, the $\Delta c_{p, a p p}$ peaks appear centred at about $20 \mathrm{~K}$ below the respective $T_{g}$ f for the amorphous and semi-crystalline PLLA samples, which proves that 
the enthalpy lost during annealing at the highest undercooling investigated is completely recovered below $T_{g}$. This confirms that structural relaxation occurs at the highest undercooling via conformational rearrangements that are completely different from the ones responsible for the glass transition process. Also a recent paper on physical ageing of polymers at temperatures far below $T_{g}$ provided evidence of a mechanism different from the slow one observed close to $T_{g}$ [16]. The presence of sub- $T_{g}$ peaks is an event commonly observed in the $c_{p}$ curves of polymeric glasses physically aged tens of degrees below $T_{g}$ [17]. The temperature of the sub- $T_{g}$ peak generally increases with the annealing time, although it was found not to approach the $T_{g}$ value for progressively increasing undercooling [17].

It is worth noting that for the amorphous PLLA annealed at low $T_{a} \mathrm{~s}$, both the $c_{p \text {,app,aged }}$ and $c_{p, \text { app,unaged }}$ curves exhibit also an enthalpy recovery peak at about $338 \mathrm{~K}$ (Figure 2 ). This second $c_{p, a p p}$ overshot is ascribable to the enthalpy loss that occurs during the cooling and the heating steps at temperatures close to $T_{g}$, where the equilibration rate is high [18]. A double enthalpy recovery in different temperature ranges further attests the difference in the equilibration mechanisms at high and low undercooling.

Several studies demonstrated that the dynamics of the structural relaxation can be described by the Vogel-Fulcher-Tamman (VFT) equation, which is generally used to fit data relative to the cooperative segmental $\alpha$-relaxation, only in proximity of $T_{g}$ [19-23], or at undercooling of few degrees $[24,25]$. With decreasing the annealing temperature, deviations from the VFT law towards a smoother temperature dependence, or an Arrhenius-type behaviour, have been reported [19-23].

Also for PLLA cooperative segmental motions could be the main and more efficient mechanism for structural relaxation close to $T_{g}$ or at undercooling of few degrees. In this temperature range not far from $T_{g}$, the $\alpha$-relaxation is expected to produce high reduction in enthalpy, due to the large-scale motions that characterize it, which involve the simultaneous rearrangements of several monomeric units. Certainly the faster local motions participate in physical ageing in the whole $\left(T_{g}-T_{a}\right)$ range investigated, but in the presence of cooperative long-range conformational rearrangements, their contribution can be less important. An investigation on the equilibration time and on the ageing rate as a function of the undercooling might evidence changes in the mechanism that mainly contributes to physical ageing in PLLA. This hypothesis is however to be confirmed, and further studies on this issue are at the moment in progress.

As discussed above, Fig. 4(B) reveals that in PLLA only sub-segmental motions produce structural relaxation at undercooling higher than $55 \mathrm{~K}$ in both amorphous and semi-crystalline PLLA. This finding leads therefore to deduce that for $\left(T_{g}-T_{a}\right)$ values included between the low undercooling at which cooperative segmental motions are active and about $55 \mathrm{~K}$, rearrangements different from 
pure large-scale cooperative and pure small-scale local motions contribute to physical ageing in PLLA.

Table 1 shows that for the two semi-crystalline PLLA samples investigated, the RAF content is similar, being the respective values within the experimental error, which means that the contribution of RAF to structural relaxation is also similar. More importantly, the trend of the normalized $\triangle h_{a} / w_{M A F}$ curves displays that at undercooling higher than about $3 \mathrm{~K}$, the supposed MAF contribution to structural relaxation is higher for the sample crystallized at higher $T_{c}$, characterized by a lower $T_{g}$, and a higher mobility of the amorphous regions. This suggests that rearrangements involving chain segments of the only MAF region could facilitate structural relaxation in this temperature range, in addition to the RAF contribution, which could be similar in the two semicrystalline PLLA samples. The different MAF mobility has been suggested to arise from different crystalline morphologies, and consequently different MAF region size [12]. The rearrangements occurring only in the MAF might be intermediate between the large-scale cooperative motions, active at low undercooling, and the small-scale local motions, the only mechanism of equilibration operative at high undercooling.

This interpretation is consistent with the results obtained from previous studies on molecular mobility in semi-crystalline PLLA, performed by dielectric relaxation spectroscopy [15]. A multicomponent character was found for the secondary $\beta$-relaxation process, which resulted from the sum of three components with variable contributions as a function of crystallinity [15]. For the slowest $\beta$-relaxation, the ratio $\left(E_{\beta} / R T_{g}\right)$ is about 21 , where $E_{\beta}=60 \mathrm{~kJ} \mathrm{~mol}^{-1}$ is the activation energy [15]. This value is very close to 24 , which was found typical of the secondary Johari-Goldstein (JG) relaxation [26]. The JG process is considered a precursor of the cooperative $\alpha$-relaxation, because at temperatures lower than $T_{g}$, it involves the motion of the entire monomeric or structural unit, via simultaneous small-scale molecular rearrangements, which do not occur cooperatively with other structural segments [27-29]. Also for some polyesters, such as poly(ethylene terephthalate), poly(ethylene naphthalene dicarboxylate), poly(ethylene isophthalate) and poly(butylene isophthalate), which exhibit different $\beta$ sub-glass modes, the slowest ones were connected with the JG relaxation [30].

Thus, the existence of simultaneous non-cooperative local motions, involving the whole repeating unit or maybe slightly larger structural portions only in the MAF region, could account partially for the structural relaxation of PLLA at undercooling between about $3 \mathrm{~K}$ and $55 \mathrm{~K}$. These associated and extended local motions could be hindered in the RAF layers, due he presence of strong constraints caused by the close crystalline regions. It is worth noting that the JG process has an Arrhenius temperature dependence in the glassy state [29], which is in perfect agreement with the 
deviations from the VFT law found for progressively decreasing annealing temperatures in previous ageing studies [19-23]. Also the JG process is expected to progressively slow down by reducing the annealing temperature, so that at undercooling higher than $55 \mathrm{~K}$, exclusively local motions become the main cause for the structural relaxation in PLLA.

\section{Conclusions}

The contribution of different motions to structural relaxation of PLLA has been identified by comparing the enthalpy recovered after isochronal annealing at different temperatures for amorphous and semi-crystalline samples. Undercooling limit of about $55 \mathrm{~K}$, below which exclusively small-scale localized motions produce structural relaxation in both amorphous and semi-crystalline PLLA has been identified. In addition, motions different from pure cooperative large-scale motions and pure small-scale local rearrangements, localized in the MAF, are found to mainly contribute to structural relaxation at intermediate undercooling. These motions are suggested to be related to the JohariGoldstein process.

The study has demonstrated that in semi-crystalline PLLA, both MAF and RAF participate in structural relaxation: MAF is supposed to contributes $(i)$ close to $T_{g}$, with cooperative large-scale motions, (ii) at intermediate undercooling, with simultaneous and associated non-cooperative local motions, involving the entire structural unit, (iii) at high undercooling, exclusively with small-scale localized motions. The contribution of the RAF, which is generated by small-scale local motions in the whole undercooling range investigated, becomes recognizable by reducing $T_{a}$, in parallel with the concomitant disappearance of the cooperative segmental motions, and the progressive reduction in non-cooperative associated local motions.

\section{Acknowledgements}

This work was partially supported by the grant "Royal Society-CNR International Exchanges Award” (IES \R2\170079). 


\section{References}

[1] B. Wunderlich, Reversible crystallization and rigid-amorphous phase in semi-crystalline macromolecules, Progr. Polym. Sci. 28 (2003) 383-450.

[2] M.C. Righetti, M. Gazzano, N. Delpouve, A. Saiter, Contribution of the rigid amorphous fraction to physical ageing of semi-crystalline PLLA, Polymer 125 (2017) 241-253.

[3] J. Dobbertin, A. Hensel, C. Schick, Dielectric spectroscopy and calorimetry in the glass transition region of semi-crystalline poly(ethylene terephthalate), J. Thermal Anal. 47 (1996) 1027-1040.

[4] C. Schick, J. Dobbertin, M. Potter, H. Dehne, A. Hensel, A. Wurm, A.M. Ghoneimm S. Weyer, Separation of components of different molecular mobility by calorimetry, dynamic mechanical and dielectric spectroscopy, J. Thermal Anal. 49 (1997) 499-511.

[5] J. Ren, K. Adachi, Dielectric relaxation in blends of amorphous poly(DL-lactic acid) and semicrystalline poly(L-lactic acid), Macromolecules 36 (2003) 5180-5186.

[6] A. Schönals, Molecular Dynamics in Polymer Model Systems in: F. Kremer, A. Schönals (Eds.), Broadband Dielectric Spectroscopy, Springer-Verlag Berlin, Germany, 2003, pp. 225-293.

[7] S.M. Sarge, W. Hemminger, E. Gmelin, G.W.H. Höhne, H.K. Cammenga, W. Eysel, Metrologically based procedures for the temperature, heat and heat flow rate calibration of DSC, J. Therm. Anal. 49 (1997) 1125-1134.

[8] P. Pan, W. Kai, B. Zhu, T. Dong, Y. Inoue, Polymorphous crystallization and multiple melting behavior of poly(L-lactide): molecular weight dependence, Macromolecules 40 (2007) 6898-6905.

[9] M.J. Richardson, N.G. Savill, Derivation of accurate glass transition temperatures by differential scanning calorimetry, Polymer 16 (1975) 753-757.

[10] E. Lopez, S.L. Simon, Signatures of structural recovery in polystyrene by nanocalorimetry, Macromolecules 49 (2016) 2365-2374.

[11] M.C. Righetti, M. Gazzano, M.L. Di Lorenzo, R. Androsch, Enthalpy of melting of $\alpha^{\prime}-$ and $\alpha-$ crystals of poly(L-lactic acid), Eur. Polym. J. 70 (2015) 215-220.

[12] M.C. Righetti, D. Prevosto, E. Tombari, Time and temperature evolution of the rigid amorphous fraction and differently constrained amorphous fractions in PLLA, Macromol. Chem. Phys. 217 (2016) 2013-2026.

[13] S.E.B. Petrie, Thermal behavior of annealed organic glasses, J. Polym. Sci Part A-2: Polym. Phys. 10 (1972) 1255-1272.

[14] A.R. Bras, M.T. Viciosa, Y. Wang, M. Dionisio, J.F. Mano, Crystallization of poly(L-lactic acid) probed with dielectric relaxation spectroscopy, Macromolecules 39 (2006) 6513-6520.

[15] A.R. Bras, P. Malik, M. Dionisio, J.F. Mano, Influence of crystallinity in molecular motions of poly(L-lactic acid) investigated by dielectric relaxation spectroscopy, Macromolecules 41 (2008) 6419-6430. 
[16] N.G. Perez-De Eulate and D. Cangialosi, The very long-term physical ageing of glassy polymers, Phys. Chem. Chem. Phys. 20 (2018) 12356.

[17] A.R. Berens, I.M. Hodge, Effects of annealing and prior history on enthalpy relaxation in glassy polymers. 1. Experimental study on poly(vinyl chloride), Macromolecules 15 (1982) 756-761.

[18] S. Montserrat, Physical aging studies in epoxy resins. I. Kinetics of the enthalpy relaxation process in a fully cured epoxy resin, J. Polym. Sci. Polym. Phys. 32(1994) 509-522.

[19] D. Cangialosi, V.M. Boucher, A. Alegria, J. Colmenero, Physical ageing in polymers and polymer nanocomposites: recent results and open questions, Soft Matter 9 (2013) 8619-8630.

[20] G.B. McKenna and S.L. Simon, 50th Anniversary perspective: challenges in the dynamics and kinetics of glass-forming polymers, Macromolecules 50 (2017) 6333-6361.

[21] P.A. O'Connell, G.B. McKenna, Arrhenius-type temperature dependence of the segmental relaxation below $T_{g}$, J. Chem. Phys. 110 (1999) 11054-11060.

[22] S. L. Simon, J. W. Sobieski and D. J. Plazek, Volume and enthalpy recovery of polystyrene, Polymer, 2001, 42, 2555-2567.

[23] J. Zhao, G.B. McKenna, Temperature divergence of the dynamics of a poly(vinyl acetate) glass: Dielectric vs. mechanical behaviors, J. Chem. Phys. 136 (2012) 154901.

[24] H. Wagner, R. Richer, Dielectric relaxation of the electric field in poly(vinyl acetate): a time domain study in the range $10^{-3}-10^{-6} \mathrm{~s}$, Polymer 38 (1997) $255-261$.

[25] V.M. Boucher, D. Cangialosi, A. Alegria, J Colmenero, Time dependence of the segmental relaxation time of poly(vinyl acetate) - silica nanocomposites, Phys. Rev. E. 86 (2012) 041501.

[26] K.L. Ngai, S. Capaccioli, Relation between the activation energy of the Johari-Goldstein $\beta$ relaxation anf $T_{g}$ of glass formers, Phys. Rev. E 69 (2004) 031501.

[27] G.P. Johari, M. Goldstein, Viscous liquids and the glass transition. III. Secondary relaxations in aliphatic alcohols and other nonrigid molecules, J. Chem. Phys. 55 (1971) 2372-2388.

[28] G.P. Johari, Intrinsic mobility of molecular glasses, J. Chem. Phys. 58 (1973) 1766.

[29] K.L. Ngai, M. Paluch, Classification of secondary relaxation in glass-formers based on dynamic properties, J. Chem. Phys. 120 (2004) 857-873.

[30] A. Nogales, A. Sanz, T.A. Ezquerra, On the role of the $\beta$ process as precursor of the $\alpha$ relaxation in aromatic polyesters, J. Non-Cryst. Solids 352 (2006) 4649-4655. 